\title{
Optimization of the imaging quality of 64- slice CT acquisition protocol using Taguchi analysis: A phantom study
}

\author{
Lung Fa Pan ${ }^{\mathrm{a}, \mathrm{b}}$, Erdenetsetseg Erdene ${ }^{\mathrm{b}}$, Chun Chi Chen ${ }^{\mathrm{c}}$ and Lung Kwang Pan ${ }^{\mathrm{b}, *}$ \\ ${ }^{a}$ Department of Cardiology, Taichung Armed Forces General Hospital, Taichung, 406, Taiwan \\ ${ }^{b}$ Graduate Institute of Radiological Science, Central Taiwan University of Science and Technology, \\ Takun, Taichung 406, Taiwan \\ ${ }^{c}$ Department of Nuclear Medicine, Chung Shan Medical University Hospital, Taichung, 402, Taiwan
}

\begin{abstract}
In this study, the phantom imaging quality of 64-slice CT acquisition protocol was quantitatively evaluated using Taguchi. The phantom acrylic line group was designed and assembled with multiple layers of solid water plate in order to imitate the adult abdomen, and scanned with Philips brilliance CT in order to simulate a clinical examination. According to the Taguchi $\mathrm{L}_{8}\left(2^{7}\right)$ orthogonal array, four major factors of the acquisition protocol were optimized, including (A) CT slice thickness, (B) the image reconstruction filter type, (C) the spiral CT pitch, and (D) the matrix size. The reconstructed line group phantom image was counted by four radiologists for three discrete rounds in order to obtain the averages and standard deviations of the line counts and the corresponding signal to noise ratios $(\mathrm{S} / \mathrm{N})$. The quantified $\mathrm{S} / \mathrm{N}$ values were analyzed and the optimal combination of the four factor settings was determined to be comprised of (A) a 1-mm thickness, (B) a sharp filter type, (C) a 1.172 spiral CT pitch, and (D) a $1024 \times 1024$ matrix size. The dominant factors included the (A) filter type and the cross interaction between the filter type and CT slice thickness $(\mathrm{A} \times \mathrm{B})$. The minor factors were determined to be $(\mathrm{C})$ the spiral CT pitch and (D) the matrix size since neither was capable of yielding a 95\% confidence level in the ANOVA test.
\end{abstract}

Keywords: 64-slice CT, reconstructed imaging, optimization, ANOVA, line phantom

\section{Introduction}

In this study, the imaging quality of Computer Tomography (CT) acquisition protocol was optimized using Taguchi analysis and a line group phantom. CT has been widely applied as a vital non-invasive diagnostic tool in the medical field and in material sciences. CT has also been proven to be accurate in demonstrating anatomy and pathology due to its precise spatial resolution $[1,2]$. Most radiographers proceed to follow phantom quality assurance protocol weekly or biweekly in order to optimize the imaging quality and avoid clinical misdiagnoses. In doing so, most adopted phantoms have been commercially standardized and assembled with multiple layers in order to assure accurate spatial resolution, densitometry, and contrast sensitivity. However, most artifacts within phantoms are

\footnotetext{
* Address for correspondence: Lung Kwang Pan, Graduate Institute of Radiological Science, Central Taiwan University of Science and Technology, Takun, Taichung 406, Taiwan. Tel.: 886-920810713; Fax: 886-4-22396762; E-mail: 1kpan@ctust.edu.tw.
} 
designed for transverse (axial) scanning rather than frontal or sagittal scanning [3]. As such, many researchers have developed customized phantoms in order to fulfill the requirements of their unique interests. Yunker at al. designed a phantom for surveying liquid flow during imaging [4]. Brisse, et al. applied PMMA cylindrical phantoms with various diameters to contrast-to-noise ratio and lowcontrast detectability evaluation [5]. Yoon, et al. used an anthropomorphic lung phantom to revise CT imaging protocol [6]. In addition, Daisne, et al. used a 3D phantom to validate the integrated performance of CT, MRI, and PET scan imaging [7]. Well-designed phantoms can effectively assist radiologists in confirming the optimal settings for routine process and verifying the accuracy, reproducibility, and consistency of multiple measurements.

In this study, a line group phantom was proposed. This particular cube model could be assembled with solid water plate of any dimension in order to accurately reflect the desired geometric size. According to the Taguchi's optimal analysis, the unique design of line-paired curves could quantify the quality of CT scan images. Since live imaging systems allow for the precise and accurate diagnoses of various carcinomas and tumor tissues, rapid and simple imaging acquisition and postprocessing methods could accelerate patient examinations. In addition, the quantitative assessment of imaging quality has always been a topic of numerous discussions in many medical diagnostic facilities. Thus, an efficient method for defining imaging quality is essential.

In this work, various post processing images of a line group phantom were scanned and reconstructed using eight combinations of four factors of 64-slice CT acquisition protocol; these eight combinations were analyzed by four highly-trained radiologists in order to quantify the imaging quality. The four factors included the (A) CT slice thickness, (B) imaging reconstruction filter type, (C) spiral CT pitch, (D) and matrix size. Furthermore, unlike some optimization processes that only focus on a single or minor factor for practicality, the four factors were simultaneously considered during the optimization process using the Taguchi method. In addition, the cross-interactions between the factors were considered in order to evaluate the complexity of the optimization.

\section{Materials and methods}

\subsection{Taguchi analysis}

The Taguchi method is a very effective method for designing high-quality systems. This method exploits unique orthogonal arrays to obtain extensive factor data using only a few experiments. The obtained optimal 64-slice CT factor settings were independent of environmental conditions and other factors. A statistical analysis of variance (ANOVA) was performed to identify the factors that significantly affected the target variable. The $\mathrm{S} / \mathrm{N}$ and ANOVA analyses were combined to determine the optimal factor settings for the 64-slice CT acquisition protocol [8-10].

\subsection{Orthogonal arrays}

Unlike other analytical optimization method, the Taguchi method determines both the optimal value of a chosen factor from a finite set of analytical data and the factors that significantly affect the target variable. This method has been widely utilized in precision manufacturing $[11,12]$ and other fields $[13$, 14]. In this work, the four factors of the 64-slice CT acquisition protocol included the CT slice thickness, filter type, spiral pitch, and matrix size. Since each of these factors could be assigned to two levels, a total of sixteen $(2 \times 2 \times 2 \times 2=16)$ combinations was considered. Using the Taguchi method, the 
Table 1

A standard $\mathrm{L}_{8}\left(2^{7}\right)$ orthogonal array suggested by Taguchi; the numbers in each column indicate the experimental layouts, or levels, of the specific factors (A-D)

\begin{tabular}{llllllll}
\hline Group & $\mathrm{A}$ & $\mathrm{B}$ & $\mathrm{A} \times \mathrm{B}$ & $\mathrm{C}$ & $\mathrm{A} \times \mathrm{C}$ & $\mathrm{A} \times \mathrm{D}$ or B $\times \mathrm{C}$ & $\mathrm{D}$ \\
\hline 1 & 1 & 1 & 1 & 1 & 1 & 1 & 2 \\
2 & 1 & 1 & 1 & 2 & 2 & 2 & 2 \\
3 & 1 & 2 & 2 & 1 & 2 & 1 & 1 \\
4 & 1 & 2 & 2 & 2 & 2 & 1 & 2 \\
5 & 2 & 1 & 2 & 1 & 1 & 2 & 1 \\
6 & 2 & 1 & 2 & 2 & 2 & 2 & \\
7 & 2 & 2 & 1 & 2 & 1 & 1 & 2 \\
8 & 2 & 2 & 1 & 2 & 2 & \\
\hline
\end{tabular}

Table 2

The four factors for CT acquisition protocol, each factor has two levels as recommended by Taguchi $\mathrm{L}_{8}$ orthogonal array

\begin{tabular}{lll}
\hline Factors & Level 1 & Level 2 \\
\hline (A) Thickness $(\mathrm{mm})$ & 1 & 2 \\
(B) Filter type & Standard & Sharp \\
(C) Spiral CT Pitch & 0.641 & 1.172 \\
(D) Matrix size & $512 \times 512$ & $1024 \times 1024$ \\
\hline
\end{tabular}

samples were organized into eight groups, which were used to obtain results with the same confidence as that of a conventional thorough optimization process [15]. Table 1 presents a standard $\mathrm{L}_{8}\left(2^{7}\right)$ orthogonal array suggested by Taguchi; the numbers in each column indicate the experimental layouts, or levels, of the specific factors (A-D). Table 2 displays the factors (A-D) as (A) a slice thickness of 1 or $2 \mathrm{~mm}$, (B) a standard or sharp imaging reconstruction filter type, (C) a 0.641 or 1.172 spiral CT pitch [(table travel/rotation)/nominal slice width], and (D) a $512 \times 512$ or $1024 \times 1024$ data reconstruction matrix size.

\subsection{Analysis of variance: ANOVA}

Loss functions $(\eta)$ measures the deviations between experimental values and desired values. Taguchi recommended the use of a loss function to express the deviation of performance characteristics from desired values. Loss function value can be transformed into signal-to-noise $(\mathrm{S} / \mathrm{N})$ ratios. Performance characteristics are categorized into one of three classes, including "lower-isbetter", "higher-is-better" and "nominal-is-best". Each class is associated with a particular S/N ratio definition, which is used during the computation of optimal factor combination. Since larger $\mathrm{S} / \mathrm{N}$ ratios always correspond to better quality, regardless of category, the optimal factors values are those that yield the highest $\mathrm{S} / \mathrm{N}$ ratios [15]. Therefore, the quality of a reconstructed image of a line group phantom obtained using various CT acquisition protocols can be calculated as

$$
\eta_{i}=-1010 \mathrm{~g}\left[\frac{1}{r} \sum_{j=1}^{r} y_{i, j}^{-2}\right]
$$

where $\eta_{i}$ represents the loss function $\left(S / N\right.$ unit: $d B$ ) of the $i_{\text {th }}$ group [16]. A larger value of $\eta$ is considered preferable herein since the quality of a phantom image is categorized as "higher-is-better". In addition, $y_{i, j}$ represents the judged grade of a phantom image for the $i_{\text {th }}$ group in the $j_{\text {th }}$ trial, and $r$ represents the total number of trial repeats in each group, which equals 12 herein (the same phantom image was graded by four radiologists for three rounds, $4 \times 3=12$ ). The F-test, developed by Dr. Fisher, 
is a test based on the assumption that the variances of two sampled populations are equal. If the variances are equal, only a $5 \%$ chance that the value of $\mathrm{F}$ will exceed $\mathrm{F}_{0.05}$ (the value of $\mathrm{F}_{0.05}$ depends on the number of samples taken from each population) exists. Therefore, if $\mathrm{F}>\mathrm{F}_{0.05}$, the variance of one population is statistically probably of being larger than that of the other population [17].

\subsection{Line group phantom and 64-slice $C T$}

The line group phantom was specifically designed to evaluate the reconstructed imaging quality of the CT acquisition protocol. The acrylic (PMMA) line group phantom $\left(60 \times 30 \times 20 \mathrm{~mm}^{3}\right)$ was specifically customized in this study. The slits were precisely milled with a $0.5-$ or $0.7-\mathrm{mm}$ cutter in order to create (1) four 4-vertical-line-slit $0.5-\mathrm{mm}$ groups, (2) five 4-horizontal-line-slit $0.7-\mathrm{mm}$ groups, (3) and two inverse 0.5- or 0.7-mm V-shaped slits. Each 4-line-slit had increments of 0.5, 1.0, $1.5,2.0$, and $2.5 \mathrm{~mm}$, the depth of each 4-line-slit varied from $0.2,0.4,0.6$, to $0.8 \mathrm{~mm}$. In addition, the two inverse V-shaped slits were milled with either a 0.5 - or $0.7-\mathrm{mm}$ cutter for comparison. These unique shapes were designed to create a series of recognizable slits that could be useful in quantifying the imaging quality of the reconstructed phantom image. In this work, the purpose of the optimization process was to increase the imaging quality (i.e., to minimize the size of the artifact). Figure 1 illustrates the well-assembled phantom ready for scanning. Figure 1(A) is a detailed view of the line group phantom. As shown in Figures 1(B) and 1(C), the phantom was placed in the center of two customized PMMA plates $\left(300 \times 300 \times 20 \mathrm{~mm}^{3}\right)$ for horizontal and vertical verification, respectively. Figure 1(D) demonstrated that the line group plate phantom was assembled with several solid water plates in order to simulate an adult abdomen $\left(300 \times 300 \times 220 \mathrm{~mm}^{3}\right)$ for CT scanning. The thicknesses of the upper, middle and bottom parts of the solid water plate were equal to $8 \mathrm{~cm}, 4 \mathrm{~cm}$ and $6 \mathrm{~cm}$, respectively $[80+20$ (phantom) $+40+20$ (phantom) $+60=220 \mathrm{~mm}]$. The 64-slice CT was located at Taichung Armed Forces General Hospital; the model was a Philips brilliance CT with a Maximus Rotalix Ceramic (MRC) X-ray tube. The other factors of the axial scan protocols were preset for standard resolution $64 \times 0.625$ collimator, $0.75-\mathrm{sec}$ of rotation time, $130-\mathrm{mm}$ FOV, no DOM, $5-\mathrm{mm}$ thickness, $5-\mathrm{mm}$ increment, $120 \mathrm{kV}$ and $250 \mathrm{mAs}$. The CT acquisition protocol was revised according to the results of an abdominal 64-slice CT study conducted at the same hospital.

\subsection{Grading the phantom}

Four radiologists counted all of the visible lines in the two reconstructed phantom images (both horizontal and vertical) of the 64-slice CT scanned imaging for every group. Each reconstructed phantom image was randomly passed to the radiologists and graded blindly in order to eliminate any biased expectations. The grading was repeated three times to ensure reproducibility and consistency. Thus, twelve counts for each group were averaged in order to determine the quality of reconstructed CT images. Numerous visible lines were visible in the reconstructed CT image with the optimal factor settings.

\section{Results}

\subsection{Data analysis}

Figure 2 displays the fish-bone-plots of the average, stdev, and $\mathrm{S} / \mathrm{N}$ values against the values of the 


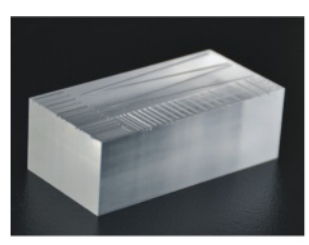

(A)

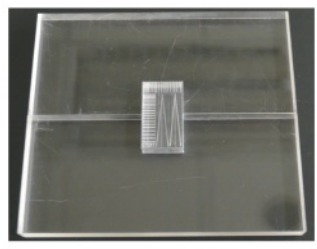

(C)

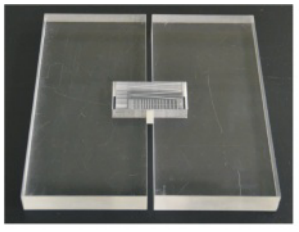

(B)

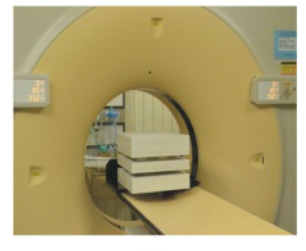

(D)
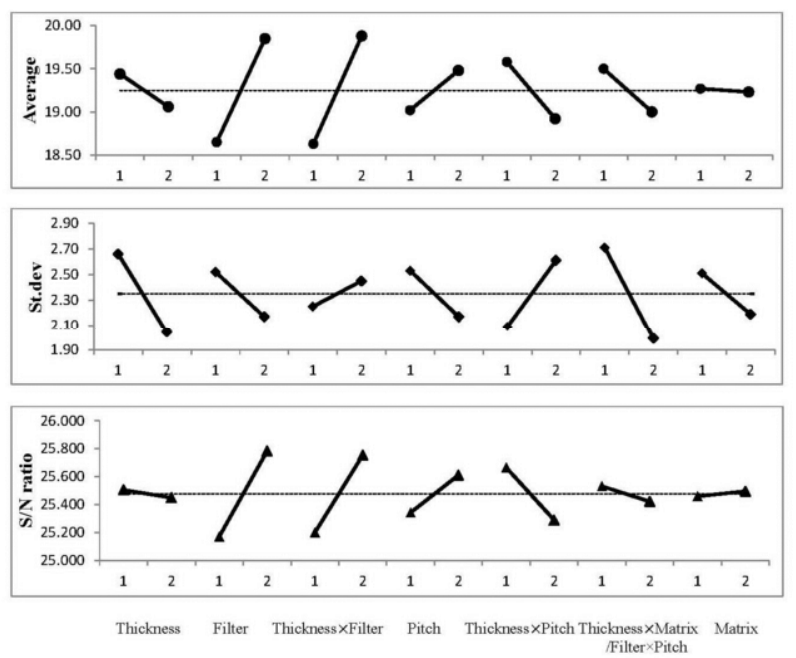

Fig. 2. The fish-bone-plots of the average, stdev and $\mathrm{S} / \mathrm{N}$ values against the values of the factors for the 64-slice CT acquisition protocol.
Fig. 1. (A) detailed view of the line group phantom, (B, C) the phantom was placed in the center of two customized PMMA plates $\left(300 \times 300 \times 20 \mathrm{~mm}^{3}\right)$ for horizontal and vertical verification, respectively, (D) the line group plate phantom was assembled with several solid water plates in order to simulate an adult abdomen $\left(300 \times 300 \times 220 \mathrm{~mm}^{3}\right)$ for CT scanning.

factors for the 64-slice CT acquisition protocol. Group 3, as indicated in Table 1, exhibited the highest values of any of the groups. The average, stdev and $\mathrm{S} / \mathrm{N}$ values for that group were equal to 20.50 , 1.98 and 26.11, respectively. Factors $B$ (filter type) and $A \times B$ (thickness $\times$ filter) were determined to be the most influential to the quality of the phantom images since the data fluctuations that were associated with the changes in these factors exceeded those associated with the changes in the other factors. Accordingly, the optimal settings of the four factors for high reconstructed imaging quality included (A) a 1-mm thickness, (B) a sharp filter type, (C) a 1.172 spiral CT pitch, and (D) a $1024 \times 1024$ matrix size (i.e., the setting of the level with a higher $\mathrm{S} / \mathrm{N}$ in each factor). The optimal settings yielded a higher image quality than the original eight groups listed in Table 1.

\subsection{Analysis of variance: ANOVA}

The identity of the dominant factors for the 64-slice CT acquisition protocol was confirmed by conducting an F-test. Table 3 presents the factor confidence levels attributed to the effectiveness of the acquisition protocol. A factor was considered significant if the corresponding confidence level exceeded 95\%. Therefore, the (B) filter type and the cross interaction between factors A and B (thickness $\times$ filter) were deemed to be significant since their associated confidence levels were equal to 95\%. Furthermore, neither factor A (thickness) nor factor B (filter type) could be adjusted individually to fulfill the specific requirement since a strong correlation existed between those two factors (Table 3). In contrast, both the spiral $\mathrm{CT}$ pitch and matrix size were deemed insignificant since their associated confidence levels were equal to $64.3 \%$ and $6.4 \%$, respectively. Therefore, no spiral CT pitch or matrix size could yield the confidence level required for a clinical diagnosis. In addition, either the spiral CT pitch or matrix size could be reasonably maintained at 1.172 or $512 \times 512$ to reduce the length of the CT scanning or post data analysis. 
Table 3

The factor confidence levels attributed to the effectiveness of the 64-slice CT acquisition protocol. A factor was considered significant if the corresponding confidence level exceeded $95 \%$

\begin{tabular}{|c|c|c|c|c|c|c|}
\hline Factor & $\mathrm{SS}$ & DOF & Variation & $\mathrm{F}$ & Confidence & Significance* \\
\hline$A$ & 3.47 & 1 & 3.47 & 0.6 & $55.4 \%$ & $\mathrm{No}$ \\
\hline$B$ & 34.56 & 1 & 34.56 & 5.8 & $98.2 \%$ & Yes \\
\hline$A \times B$ & 37.50 & 1 & 37.50 & 6.3 & $98.6 \%$ & Yes \\
\hline C & 5.08 & 1 & 5.08 & 0.9 & $64.3 \%$ & No \\
\hline$A \times C$ & 10.45 & 1 & 10.45 & 1.8 & $81.3 \%$ & No \\
\hline$A \times D \quad$ or & 6.00 & 1 & 6.00 & 1.0 & $68.3 \%$ & No \\
\hline$D$ & 0.04 & 1 & 0.04 & 0.0 & $6.4 \%$ & No \\
\hline Total & 618.00 & 95 & *At least & confidence & & \\
\hline
\end{tabular}

\section{Discussion}

\section{1. $L_{8}$ orthogonal array}

The Taguchi method not only yielded the dominant factor, but also effectively elucidated the individual cross-interactions among the factors. In the unique orthogonal arrangements of the various factor values, the frequencies of the levels were constant for all eight groups (Table 1); the obtained data was rearranged to elucidate the cross-interactions among the factors. This particular data arrangement was critical in analyzing the $\mathrm{L}_{8}\left(2^{7}\right)$ orthogonal array because it provided a regulated basis for compiling three cross interactions among four individual factors; thus as indicated by Taguchi, it only supported level-IV resolution since the cross-interactions among the factors could conflict together (Table 1, column 6) [15].

Figure 3 presents four cross interactions between pairs of factors. Parts (A), (B), (C), and (D) of Figure 3 display the cross interactions between filter vs. thickness $(A \times B)$, pitch vs. thickness $(A \times C)$, matrix vs. thickness $(A \times D)$, and pitch vs. filter $(B \times C)$, respectively, for the 64-slice $C T$ acquisition protocol (Table 1, columns 3,5, and 6). Three of the four cross interactions among the factors

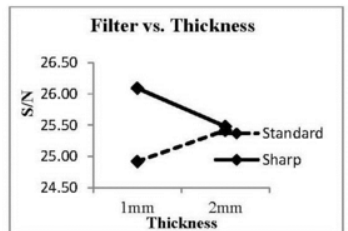

(A)

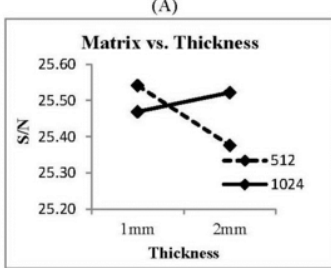

(C)
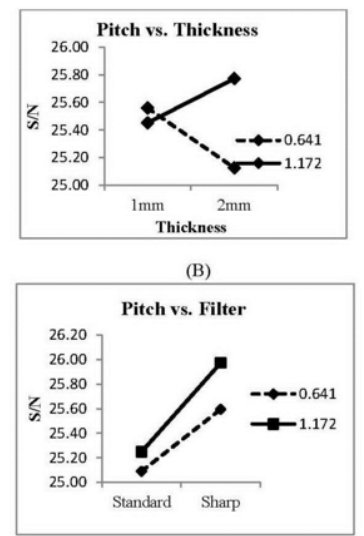

Fig. 3. Four cross interactions between pairs of factors. Parts $(A),(B),(C)$, and (D) filter vs. thickness $(A \times B)$, pitch vs. thickness $(A \times C)$, matrix vs. thickness $(A \times D)$, and pitch vs. filter $(B \times C)$, respectively, for the 64-slice $C T$ acquisition protocol (Table 1 , columns 3,5 , and 6 ). 
exhibited strong interactions, indicating that the values for factors C (spiral CT pitch) and D (matrix size) had to be switched with those of either factor A (thickness) in level one or factor B (filter type) in level two in order to optimize the performance. In addition, factor B (filter type) dominated all of the other factors. Neither $\mathrm{A} \times \mathrm{D}$ nor $\mathrm{B} \times \mathrm{C}$ dominated the $\mathrm{CT}$ imaging quality since their confidence levels only reached $68.3 \%$ (Table 3 ); however, $\mathrm{A} \times \mathrm{D}$ exhibited a strong interaction in actuality.

\subsection{Definition of $S / N$}

The definition of S/N (Eq. (1)) adopted herein could be revised to satisfy different requirement. Eq. (2) defines the $\mathrm{S} / \mathrm{N}$, which integrates the average grade and standard deviation in order to yield a low statistical fluctuation, as [18],

$$
\eta_{i}=-10 \log \left[\left(\begin{array}{ll}
\text { Stdev } & i / \hat{y}_{i}
\end{array}\right)^{2}\right]
$$

where $\overline{y_{i}}$ and $\operatorname{stdev}_{\mathrm{i}}$ represent the average grade and statistical deviation of the $\mathrm{i}_{\text {th }}$ group. The recalculated $\mathrm{S} / \mathrm{N}$ values for groups one through eight were equal to $15.35,17.83,2031,16.31,16.04$, $21.28,19.18$, and 22.27, respectively (Eq. (2)). The highest $\mathrm{S} / \mathrm{N}$ value was originally observed in group three, but was observed in group eight of the reconstructed images. Furthermore, of the dominant factors, the filter type (B) was sharp for both group three and group eight, but the thickness (A) changed from 1-mm to $2-\mathrm{mm}$ in order to maintain a low statistical fluctuation. However, this recommended CT acquisition protocol optimization was compromised in that focusing on only one quality characteristic could have altered the results.

\section{Conclusion}

The imaging quality of a 64-slice CT of a reconstructed phantom was optimized using Taguchi analysis and a line group phantom. A PMMA line group phantom was precisely milled to form groups of 0.5- or 0.7-mm-wide slits with various depths and increments in order to successfully optimize the reconstructed imaging quality achieved during routine diagnostic protocol. Eight combinations of the values of four factors of the 64-slice CT acquisition protocol were determined using the Taguchi method. The optimal factor settings included (A) a 1-mm thickness, (B) a sharp filter type, (C) a 1.172 spiral CT pitch, and (D) a $1024 \times 1024$ matrix size; these optimal setting yielded the highest line count average and $\mathrm{S} / \mathrm{N}$ ratio. The ANOVA analysis revealed that the dominant factors were the filter type and the cross interaction between filter type and thickness. The minor factors included the spiral CT pitch and the matrix size of the 64-slice CT acquisition protocol.

\section{Acknowledgment}

The authors would like to thank the Ministry of Science and Technology, contract No. 101-2221-E166-001 and the Taichung Armed Forces General Hospital, Contract No. 102A08 for financially supporting this research.

\section{References}


[1] H. Watanabea, E. Hondab, A. Tetsumuraa, et al., A comparative study for spatial resolution and subjective image characteristics of a multi-slice CT and a cone-beam CT for dental use, European Journal of Radiology 77 (2011), 397402.

[2] F. Zarb, L. Rainford and M.F. McEntee, Developing optimized CT scan protocols: Phantom measurements of image Quality, Radiography 17 (2011), 109e114.

[3] Catphan ${ }^{\circledR}$ Phantoms, available at: http://www.phantomlab.com/products/catphan.php, last accessed: Jun. $2^{\text {nd }}, 2015$.

[4] B.E. Yunker, D. Cordes, A.L. Scherzinger, et al., An investigation of industrial molding compounds for use in 3D ultrasound, MRI, and CT imaging phantoms, Medical Physics 40 (2013), 052905.

[5] H.J. Brisse, J. Brenot, N. Pierrat, et al., The relevance of image quality indices for dose optimization in abdominal multi-detector row CT in children: Experimental assessment with pediatric phantoms, Physics in Medicine and Biology 54 (2009), 1871-1892.

[6] S. Yoona, R.W. Henry, D.M. Bouley, et al., Characterization of a novel anthropomorphic plastinated lung phantom, Medical Physics 35 (2008), 5934-5943.

[7] J.F. Daisnea, M. Sibomanab, A. Bolb, et al., Evaluation of a multimodality image (CT, MRI and PET) coregistration procedure on phantom and head and neck cancer patients: Accuracy, reproducibility and consistency, Radiotherapy and Oncology 69 (2003), 237-245.

[8] C.Y. Chen, K.C. Liu, H.H. Chen et al., Optimizing the TLD-100 readout system for various radiotherapy beam doses using the Taguchi methodology, Applied Radiation and Isotopes 68 (2010), 481-488.

[9] L.K. Pan, D.S. Chou and B.D. Chang, Optimization for solidification of low-level- radioactive resin using Taguchi analysis, Waste Management 21 (2001), 762-772.

[10] L.K. Pan, C.C. Wang, Y.C. Hsiao et al., Optimization of Nd: YAG laser welding onto magnesium alloy via Taguchi analysis, Optic and Laser Technology 37 (2004), 33-42.

[11] W.H. Yang and Y.S. Tarng, Optimization of the weld bead geometry in gas tungsten arc welding by the Taguchi method, International Journal of Advanced Manufacturing Technology 14 (1998), 549-554.

[12] S. Miyazawa, N.H. Loh and S.C. Tam, A study of the effects of ball burnishing factors on surface roughness using factorial design, Journal of Mechanical Work Technology 18 (1989), 53-61.

[13] A.S. Al-Bsharat and A.M. Hassan, Improvements in some properties of non-ferrous metals by the application of the ball burnishing process, Journal of Material Processing Technology 59 (1996), 250-256.

[14] S. Miyazama, N.H. Lon and S.C. Tam, Application of experimental design in ball burnishing, International Journal of Machine Tools and Manufacture 33 (1993), 841-852.

[15] G. Taguchi, Introduction to quality engineering, Asian Productivity Organization Tokyo, 1990.

[16] M.S. Phadke, Quality Engineering Using Robust Design, Prentice Hall, Englewood Cliffs, New Jersey, 1989, p. 250.

[17] R.A. Fisher, Design and Analysis of Experiments, Olive and Boyd, London, 1925.

[18] D.M. Yeh, P.J. Chang and L.K. Pan, The optimum Ga-67-citrate gamma camera imaging quality factors as first calculated and shown by the Taguchi's analysis, Hellenic Journal of Nuclear Medicine 16 (2014), 25-32. 